\title{
CALCIUM AND IRON ABSORPTION: INVITRO STUDIES
}

\section{Bharat Kwatra}

Invezion labs, Conceptive Farmacia

Article Info: Received 20 November 2019; Accepted 10 December 2019

DOI: https://doi.org/10.32553/ijmbs.v3i12.801

Corresponding author: Bharat Kwatra

Conflict of interest: No conflict of interest.

\section{Abstract}

Studies on human subjects have shown that $\mathrm{Ca}(\mathrm{Ca})$ will inhibit iron (Fe) absorption, regardless of whether or not it's given as Ca salts or in dairy farm products. This has caused concern as increased Ca intake usually is usually recommended for kids and girls, similar populations that are in danger of metal deficiency. However, a radical review of studies on humans during which $\mathrm{Ca}$ intake was considerably increased for long periods shows no changes in hematologic measures or indicators of iron standing. Thus, the repressing impact additionally be|is also of short period and there also is also counteractive mechanisms. The interaction between Ca and metal is also a lumenal event, poignant metal uptake through DMT1 (divalent metal transporter 1) at the top membrane. However, it's additionally attainable that inhibition happens throughout metal transfer into circulation, suggesting roles for the serosal bourgeois ferroportin (FPN) and hephaestin. We explored these prospects in human enteral Caco-2 cells cultivated in monolayers. Iron transport (fifty nine $\mathrm{Fe}$ ) and expression of DMT1, FPN, and hephaestin were assessed when one.5 and four hours with zero or a hundred $\mu \mathrm{M} \mathrm{CaCl} 2$. though $\mathrm{Ca}$ failed to have an effect on metal uptake or DMT1 expression at one.5 hours, FPN abundance at the basolateral membrane remittent, leading to increased cellular metal retention and remittent metal efflux. when four hours, DMT1 and FPN expression increased and there was an increased FPN.

\section{Introduction}

The adverse effects of iron deficiency anemia and iron deficiency area unit standard and interventions victimization iron fortification or supplements are implemented to prevent iron deficiency. However, even though such strategies could also be successful partially, it's evident that a common downside is that the presence of dietary factors, such as phytate and polyphenols, that inhibit iron absorption. Any a $p$ approach to enhance the iron standing of vulnerable populations ought to, therefore, embrace the consideration of limiting the intake of such restrictive factors. Calcium has been found to possess a negative impact on iron absorption in humans [one - six]. Over a spread of "physiological" atomic number 20 intakes, iron absorption was reciprocally correlative to the atomic number 20 content of the meal (5). Similar effects were determined for atomic number 20 salts (used for supplements) and milk/dairy products. a lucid answer to the present organic process downside would be to limit the atomic number 20 content of iron-containing meals so iron absorption is optimized
[7]. However, this strategy ends up in concern about atomic number 20 nutrition. it's standard that the same population teams that area unit prone to iron deficiency; i. e., infants, children, and girls of childbearing age, even have high necessities for atomic number 20. Indeed, the foremost recent DRIs [Dietary Reference Intakes] for atomic number 20 was I rumpled as a consequence of findings on the health benefits of high atomic number 20 intake [8]. limiting the atomic number 20 content of 1 or two of the key meals of the day creates goodish difficulties to attain the suggested daily intakes of atomic number 20. for instance, a young teenage girl WHO is usually recommended to consume one hundred zero mg of calcium per day, might commonly take this in throughout three meals (breakfast, lunch, dinner) and a sizeable snack, that interprets to a mean of twenty-five zero $\mathrm{mg}$ per meal. If 2 of those meals area unit presupposed to be low in atomic number 20 (to improve iron absorption), the other two meals ought to contain fifty zero $\mathrm{mg}$ per meal. This is difficult to attain with "normal" food elements and will possibly necessitate atomic number 20 fortified 
ion and/or atomic number 20 supplementation. This, in turn, will require associate awareness of the buyer to a) choose high-calcium diets for a few meals and b) to pick out iron-containing foods at bound meals, whereas limiting calcium intake from constant meals. From a health education purpose of reading, this can be a fancy and difficult message to convey. Before making an attempt to revise dietary pointers and their implementations (school lunch programs, food provisions, etc.), it seems affordable to explore whether or not acute effects of atomic number 20 or iron absorption cause any negative long-run effects on iron standing with calcium in a tablet.

\section{Method and Discussion}

We have investigated the potential adaptation of iron absorption to high calcium concentrations using the human intestinal Caco-2 cell line (Lonnerdal et al., to be submitted). Briefly, Caco-2 cells were grown in Transwell chambers for 14 days until post-confluent. The formation of monolayers was confirmed by measuring transepithelial electrical resistance (TEER). Medium in the upper well (apical side) was replaced with serum-free medium (SFM) containing $1 \mu \mathrm{M}$ FeSO 4 (labeled with $59 \mathrm{Fe}$ ) together with 0 or $100 \mu \mathrm{M} \mathrm{CaCl}$ 2. After 1.5 and 4 hours, iron $(59 \mathrm{Fe}$ ) in the top (apical) chamber and the bottom (basolateral) compartment was measured by gamma counting. Effects of $\mathrm{Ca}$ on

DMT1 expression (apical Fe uptake), FPN expression and localization by antibody capture (serosal Fe Efflux), and hephaestin expression and activity (Fe oxidation) were determined. DMT1 and FPN gene expression were measured by quantitative real-time polymerase chain reaction (QRT-PCR) and protein expression by Western blotting as described earlier [7]. Hephaestin activity was analyzed by an in-gel colorimetric assay and using densitometry to quantitate any significant differences. We found a significant reduction in base lateral transfer of iron after both 1.5 and 4 hours and slightly more iron in the apical chamber after 1.5 hours.

Thus, there was an initial inhibitory effect of calcium on iron transfer across the epithelial cell, which most likely would cause an effect on iron absorption measured in single-meal studies, as iron largely has been absorbed within 4 hours after a meal. The expression of DMT1 was slightly reduced $(p=0.06)$ after 1.5 hours, but after 4 hours, it was significantly increased (Figure 1). There was no effect on total FPN after 1.5 hours, but surface-bound FPN was significantly decreased, suggesting re-localization of membrane-bound FPN into an intracellular compartment. It is well-known that the localization and therefore the function of FPN can be modulated by various conditions [8]. After 4 hours, FPN expression had increased slightly $(p=0.06)$ and more FPN were now located on the basolateral membrane (Figure 2). There was no significant effect of calcium on hephaestin expression or activity (data not shown). Although these studies need to be extended in time, it is apparent that there is an initial inhibitory effect of iron transfer across the intestinal cell and o that this is due to a transitory re-localization of FPN into an intracellular location, where it cannot transport iron. With time, FPN is transported back to the base o lateral membrane, which should lead to a compensatory increase in iron than sport from the cell to the systemic circulation. This may be accompanied by some down- and up-regulation of DMT1 and FPN. Thus, iron homeostasis may be achieved in dynamic changes in iron transporter localization and possibly expression. Since calcium inhibits the absorption of both heme and nonheme iron [5] and these two forms of iron are taken up at the apical membrane by two very different transporters, HCP1 and DMT1, respectively, we found it likely that the calcium effect would be exerted post-uptake; i. e. when iron released from heme by heme oxygenase intracellularly and ferrous iron transferred by DMT1 have formed a common iron pool. We, therefore, studied both FPN expression and its membrane localization. Recently, Thompson et al.

[9] performed experiments similar to ours also using Caco-2 cells in monolayers. They found that the ironinduced increase in cellular ferritin was decreased by the addition of calcium $(2.5 \mathrm{mM})$. The localization of DMT1 changed, in that this transporter became intracellular when exposed to high calcium concentrations, but there was no change in total DMT1. Since these authors did not study FPN localization and we did not study DMT1 localization it is possible that both these events occurred in response to increased calcium exposure. An isolated effect on DMT1 localization, however, would not explain the effect of calcium on heme-iron absorption as DMT1 does not transport this form of iron.

\section{Results and Conclusion}

Calcium has been shown to affect iron absorption in some short-term studies In those studies, in which an effect has been observed, the magnitude of the effect 
varies considerably and appears to be dependent on the methodology was chosen. the total absence of any negative effect of iron status of vulnerable groups fed high levels of calcium.

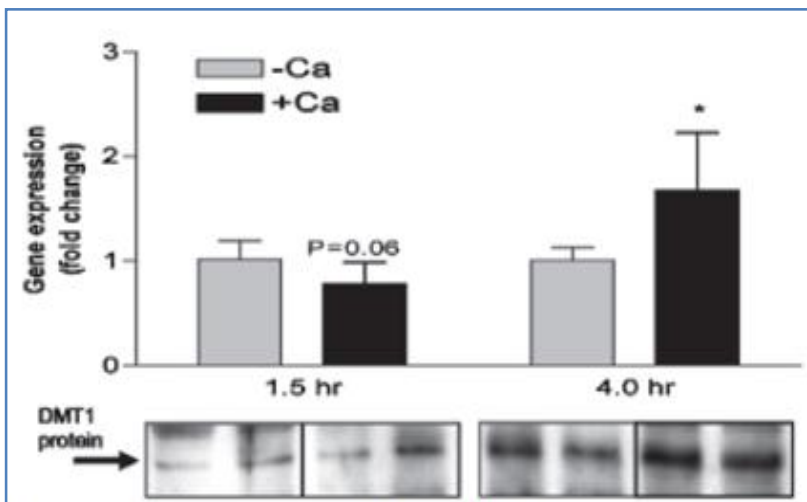

Figure 1: Expression of DMT1 mRNA (top panel) determined by QRT-PCR and protein (bottom panel) determined by Western blot (duplicate samples; left side, left panel - Ca, right panel at $1.5 \mathrm{~h}$; right side, left panel $-\mathrm{Ca}$, right panel at $4.0 \mathrm{~h})$.

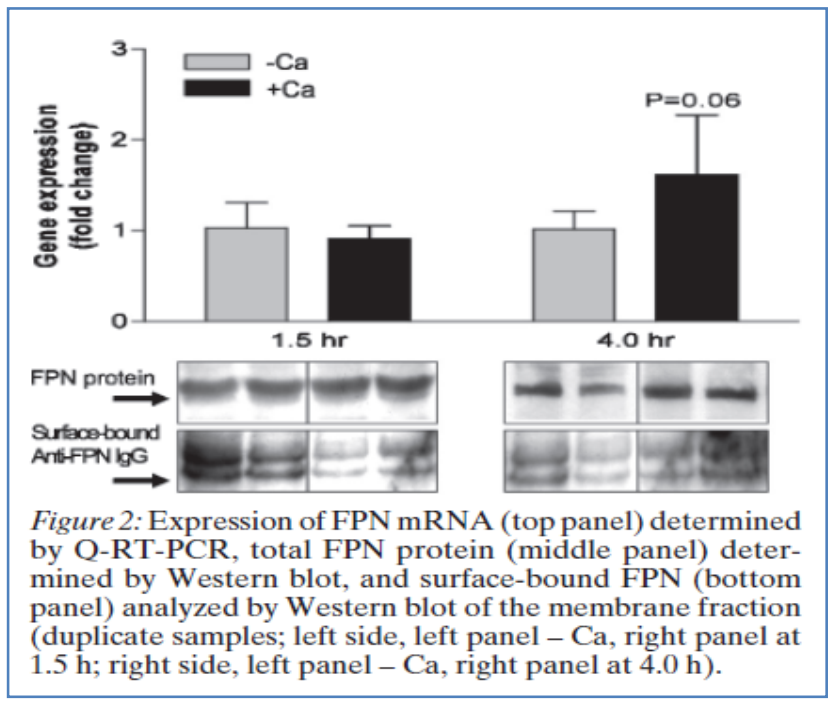

\section{References}

1. Monsen, E.R. and Cook, J.D. (1976) Food iron absorption in human subjects IV. The effects of calcium and phosphate salts on the absorption of nonheme iron. Am. J. Clin. Nutr. 29, 1142.

2. Dawson-Hughes, B., Seligson, F.H. and Hughes VA. (1986) Effects of calcium carbonate and hydroxyapatite on zinc and iron retention in postmenopausal women. Am. J. Clin. Nutr. 44, 83.

3. Deehr, M., Dallal, G.E., Smith, K.T., Taulbee, J.D. and Dawson-Hughes, B. (1990) Effects of different calcium sources on iron absorption in postmenopausal women. Am. J. Clin. Nutr. 51, 95.

4. Cook, J.D., Dassenko, S.A. and Whittaker, P. (1991) Calcium supplementation: effect on iron absorption. Am. J. Clin. Nutr. 53, 106.

5. Hallberg, L., Brune, M., Erlandsson, M., Sandberg, A.S. and Rossander-Hulten, L. (1991) Calcium: effect of different amounts of non-haem- and haem-iron absorption in humans. Am. J. Clin. Nutr. 53, 112.

6. Hallberg, L., Rossander-Hulten, L., Brune, M. and Gleerup, A. (1992) Bioavailability in man of iron in human milk and cow's milk in relation to their calcium content. Pediatr. Res. 31, 524.

7. Hallberg, L., Rossander-Hulten, L. and Gleerup, A. (1992) Calcium and iron absorption: mechanisms of action and nutritional importance. Eur. J. Clin. Nutr. 26, 317.

8. Leong, W.I., Bowlus, C.L., Tallkvist, J. and Lönnerdal, B. (2003) Iron supplementation during infancy-effects on expression of iron transporters, iron absorption, and iron utilization in rat pups. Am. J. Clin. Nutr. 78, 1203.

9. Wolff, N.A., Liu, W., Fenton, R.A., Lee, W.K., Thévenod, F. and Smith, C.P. ( 2009) Ferroportin 1 is expressed basolaterally in rat kidney proximal tubule cells and iron excess increases its membrane traffi cking. J. Cell Mol. Med. Dec 8. [Epub ahead of print] 